\title{
APPRENTISSAGE DU FLE EN LITUANIE ET MOTIVATION
}

\author{
Dr. Miroslav Stasilo \\ Lietuvos karo akademija \\ Vilniaus universitetas
}

Sommaire. L'Europe est caractérisée par le multilinguisme depuis les temps les plus anciens jusqu'à maintenant. Le plurilinguisme et le multiculturalisme, c'est la base et la particularité de l'identité européenne. La politique linguistique des institutions européennes occupe une place importante en Europe et notamment en Lituanie où la moyenne des gens maitrisant deux ou trois langues étrangères est plus élevée que celle dans l'UE. Or, le nombre de francophones ici est moins enlevé que la moyenne européenne.

Il se présente avantageux d'examiner le point de vue des étudiants lituaniens de l'Académie militaire de Lituanie, de l'université de Vilnius et du Centre de la formation des douaniers concernant leur motivation d'apprendre le français qui est un facteur décisif lors du processus éducatif. La desription et l'analyse des réponses des enquêtes liées à l'apprentissages du français ou d'autres langues dans des universités, l'Académie et un centre de la formation en Lituanie permet de dévoiler les facteurs principaux du choix linguistique des étudiants lituaniens.

L'enseignant moderne doit tout d'abord créer des conditions favorables à l'apprentissage en aidant l'apprenant à attendre ses objectifs. Il y a un grand choix de méthodes et de possibilités pour apprendre le français langue étrangère, par ex., l'enseignement hybride, la mémorisation verbale ou des activités théâtrales. Le but principal de toutes ces approches est de soutenir l'autonomie, la réflexion logique, l'intégration et la participation active des apprenants. Le processus éducatif moderne envisage une collaboration et une interaction active entre l'enseignant et l'apprenant. L'enseignant de français, en se basant sur des méthodes actives et sa créativité, doit prendre en compte les différences concernant le niveau linguistique, l'expérience et l'autonomie des individus ainsi que les objectifs communs et personnels.

Mots-clès : multilinguisme, FLE, motivation, apprentissage actif, enseignement hybride, activités théâtrales.

\section{Introduction}

Actualité et problématique. On distingue trois grands centres économiques, politiques et culturels dans le monde moderne : États-Unis, Chine et Europe. Historiquement, les États-Unis sont dominés par deux langues - l'anglais et 
l'espagnol. La Chine favorise le développement et la popularisation de la langue chinoise écrite standard (mandarin), désignée comme "la langue véhiculaire de la nation", surtout à partir de l'arrivée au pouvoir du parti communiste dans les années 50-60 (Minglang Zhou, 2003 : 401). Etant donné la diversité linguistique un élément clef de l'identité et de la culture européenne, les institutions de l'UE à Bruxelles et à Strasbourg, notamment le Conseil de l'Europe et le Conseil européen, encouragent l'apprentissage des langues vivantes dont le français langue étrangère (FLE). Le sujet traité nous aidera à présenter la spécificité du travail de l'enseignant du FLE en Lituanie et à voir les raisons ainsi que les motivations des apprenants de l'Académie militaire de Lituanie (AML), de l'université de Vilnius (UV) ou du Centre de la formation des douaniers (CFD) d'évoluer en développant leurs compétences linguistiques en plusieurs langues dont le français.

Notre objet de recherche se concentre sur l'enseignement ainsi que l'apprentissage du FLE en Lituanie, qui est en accord avec les lignes directrices concernant la politique linguistique de l'UE. Le français n'est pas la première langue vivante enseignée en Europe et nous nous intéressons par conséquent aux raisons ainsi qu'aux motifs des apprenants d'étudier cette langue. Notre étude est axée sur les observations et réflexions liées à l'apprentissage ou l'enseignement du FLE dans le cadre de l'enseignment formel dans des universités lituaniennes et dans le cadre de la formation continue professionnelle au CFD.

Notre but - répondre aux questions : " Quelles sont les méthodes d'enseignement des langues étrangères aujourd'hui les plus efficaces ? Quel est le statut de l'enseignant dans le processus éducatif actuel ? Quelles sont les raisons et motivations d'apprendre une langue vivante? Quelle est l'importance de l'approche active et quelle est la place du lexique ainsi que des activités théâtrales dans une telle approche? ».

Méthodes. Nos nous basons sur des théories psychologiques ou psychoéducologiques issues des ouvrages de R. Atkinson, D. Beresnevičiené, G. Butkienè, Z. Dörnyei, H. Ebbinghaus, N. Gage, R. C. Gardner, T. Hatch, L. Jovaiša, A. Kepalaite, G. Petty, F. Rheinberg, R. M. Shiffrin, C. Tavris, etc., ainsi que sur les données statistiques du Département de statique de la République de Lituanie, de l'AML, de l'UV et de l'AML. Nous appliquons la méthode descriptive, comparative et statistique à nos recherches.

\section{Politique linguistique de l'UE}

La Lituanie fait partie de l'UE et respecte les directives provenant du Conseil de l'Europe qui a reconnu et validé le Cadre européen commun de référence pour les langues (CECR) (angl. Common European Framework for Languages) en 2001 afin de réaliser et mettre en oeuvre la politique de l'enseignement, de l'apprentissage et de l'évaluation des langues étrangères au sein de l'Europe. Ce document, fruit d'un grand travail, a été mené depuis 1991 par plusieurs représentants de la profession 
enseignante à travers de l'Europe et au-delà. Le CECR a élaboré de nouveaux programmes et des manuels pour les langues vivantes avec des tests et examens en unifiant la grille d'évaluation. Le CECR décrit un certain nombre de compétences linguistiques pour que l'apprenant ainsi que l'enseignant définissent leurs objectifs et améliorent le savoir-faire en langue vivante pour la communication quotidienne et la vie professionnelle. C'est aussi la raison pour laquelle le CECR introduit le contexte culturel dans le processus éducatif.

Le CECR n'est pas le premier modèle qui décrit les niveaux de connaissances linguistiques. Dans la deuxième moitié du siècle dernier, les enseignants ont compris que les méthodes traditionnelles d'apprentissage, quand il fallait apprendre par cœur beaucoup de règles et de nouveaux mots, traduire des textes, surtout littéraires, n'étaient plus opérationnelles (Rosen, 2010). A la fin des années 70, de nouvelles méthodes communicatives, qui mettent accent sur l'action et le contexte, en y intégrant des connaissances culturelles, la kinésique et des situations de communication, se sont apparues. Les apprenants ne devaient plus apprendre seulement à conjuguer correctement les verbes, à utiliser les articles ou à faire des listes de mots organisés autour de thèmes (animaux, travail, maison, etc.) mais ils devaient aussi savoir se présenter, échanger des opinions, des sentiments, bâtir des hypothèses, porter un jugement, etc. Les méthodes communicatives utilisent des documents authentiques, par exemple, une fiche d'inscription pour un cours de langues ou des extraits d'un journal télévisé, ainsi que des documents crées, par ex., un dialogue ou un texte d'un manuel, dans le but de présenter la vie réelle et de faire apprendre comment il faut communiquer, se comporter et réagir.

Les compétences communicationnelles permettent de devenir plus

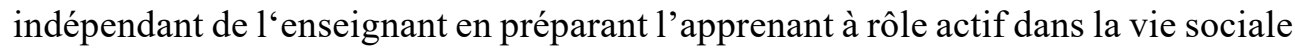
grâce à la modélisation des situations concrètes via une langue étrangère. Cela nécessite que l'enseignant soit plus créatif dans l'élaboration et la présentation des programmes d'apprentissage. Il faut aussi que l'apprenant interagisse plus courageusement et avec plus d'enthousiasme dans des situations et activités proposées par l'enseignant.

\section{Apprentissage hybride en français au CFD}

Pour répondre aux besoins des apprenants, les andragogues doivent diversifier leurs méthodes de travail dont l'une des possibilités est l'approche hybride (kombinuotas mokymas en lituanien ou Blended Learning en anglais) qui se focalise sur le paradigme d'apprentissage (voire de transaction) dont la conception de l'enseignement est le développement des connaissances avec des réponses aux questions complexes et la création de relations juxtaposées.

Ce paradigme met l'accent sur des schémas cognitifs en recherchant des réponses aux questions personnelles. L'enseignant organise le processus éducatif et crée des activités à partir de l'apprenant qui fait des recherches, rencontre des 
situations problématiques ou développe des projets. L'évaluation personnelle des apprenants se fait en se basant sur les références liées aux compétences développées par des portfolios, des tâches intégratrices et moins sur les résultats des tests à choix multiple. L'andragogue y joue un rôle important du guide qui peut aider ou donner des conseils. Il peut communiquer avec les apprenants soit sur place, quand la formation se déroule dans l'établissement qui organise la formation, soit à distance, quand les participants se forment en ligne. Ce qui est le plus important ici, c'est une interaction mutuelle entre l'andragogue et un apprenant adulte. L'andragogue donne des moyens de constituer un bagage personnel d'outils linguistiques pour atteindre les objectifs d'apprentissage. Cela permet de renforcer la conviction de l'apprenant que sa découverte a été signifiante car elle fait partie d'un système organisé où la synthèse et la création appartiennent à la perception cognitive la plus haute selon la taxonomie de Bloom dont on a parlé auparavant. Le CFD à Vilnius est l'un des premiers établissements d'éducation non formelle du pays qui a employé cette méthode novatrice afin de favoriser l'apprentissage tout au long de la vie.

Ce type d'apprentissage a eu un grand succès auprès des apprenants. Cela est prouvé par les réponses anonymes du questionnaire distribué à la fin des cours. L'enquête de satisfaction comporte 11 questions : «Comment évaluez-vous les cours ? », «Quelles étaient vos attentes avant de participer aux cours du CFD ? », « Les cours ont-ils satisfait vos attentes?», "Avez-vous lu le programme d'apprentissage ? », « Est-ce que tous les thèmes étaient utiles pour vous ? », « Quels thèmes vous ont plu le plus ? », «Quels thèmes pourrait-on supprimer? », «Qu'est-ce qu'on pourrait y ajouter?», «Comment évaluez-vous le rythme et l'intensivité des cours ?», « Quelles seraient vos propositions pour l'enseignant ? » et « Envisagez-vous d'appliquer les connaissances acquises dans votre travail ?».

Si nous comparons les réponses après les cours en présentiel et celles basées sur l'apprentissage hybride en français, nous constatons la satisfaction plus élevée pour l'approche hybride : 76 douaniers ont donné la meilleure note (« très bien »), 8 personnes ont répondu « bien » et ce ne sont que 2 participants qui ont écrit « note moyenne » pour ce type de cours (cf. Graphique $n^{\circ} 1$ ). Quatre-vingt-douze douaniers des cours en présentiel ont répondu au même questionnaire. Les réponses indiquent que 58 douaniers ont donné la meilleure note, 21 personnes - « bien », 11 apprenants - « note moyenne », 2 personnes - « mauvaise note » (ibidem). 


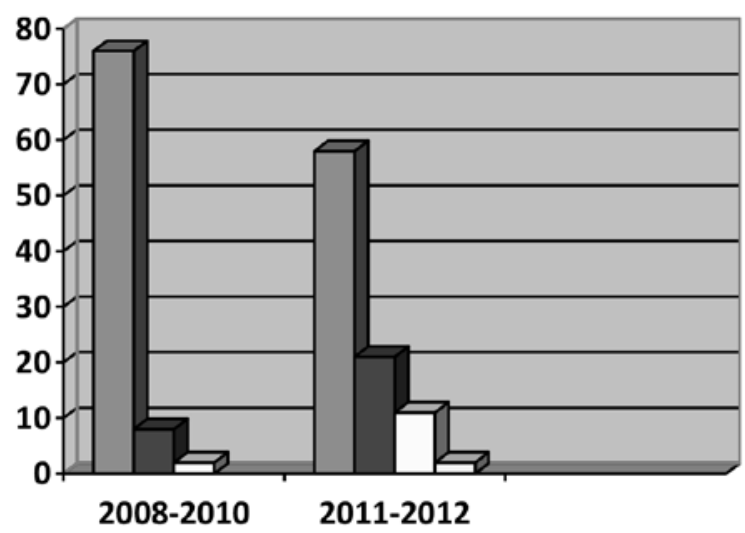

\begin{tabular}{|l|}
\hline$\square$ Très bien \\
$\square$ Bien \\
$\square$ Note moyenne \\
$\square$ Mauvaise note \\
\hline
\end{tabular}

Graphique $^{\circ}{ }^{1}$. Satisfaction des cours (2008-2012)

Concernant le rythme, entre 2008 et 2010, 23 douaniers ont estimé celui des cours traditionnels trop intensif, 31 - intensif, 36 - normal, 2 - trop lent (cf. Graphique $\mathrm{n}^{\circ} 2$ ) ; entre 2011 et 2012, 14 douaniers ont répondu que le rythme était trop intensif, 38 - intensif, tandis que 34 ont suggéré qu'il était normal (ibidem).

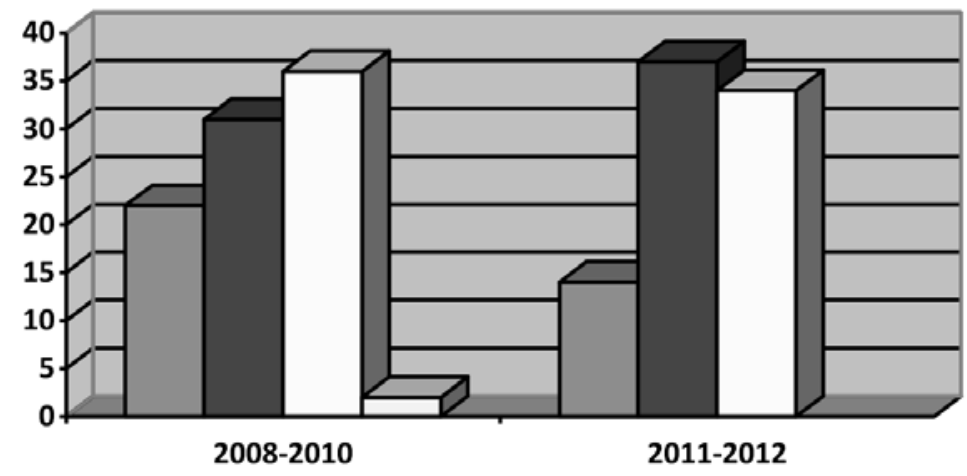

\begin{tabular}{|l|}
\hline Trop intensif \\
$\square$ Intensif \\
$\square$ Normal \\
$\square$ Lent \\
\hline
\end{tabular}

Graphique n 2. Rythme des cours (2008-2012)

La dernière question portait sur l'utilité pratique des cours : entre 2011 et 2012, 2 personnes ont répondu qu'elles ne voyaient pas d'utilité des connaissances linguistiques acquises, 35 participants ont noté qu'ils allaient parfois employer leur bagage linguistique au travail et 70 douaniers voulaient l'appliquer immédiatement au travail (cf. Graphique $\mathrm{n}^{\circ} 3$ ) ; entre 2008 et 2010, 11 personnes ne voyaient pas d'utilité des connaissances acquises, 14 participants allaient parfois employer leur bagage linguistique et 66 douaniers ont prévu d'avoir recours à leurs connaissances, reçus grâce à ces cours, quotidiennement au travail (ibidem). 

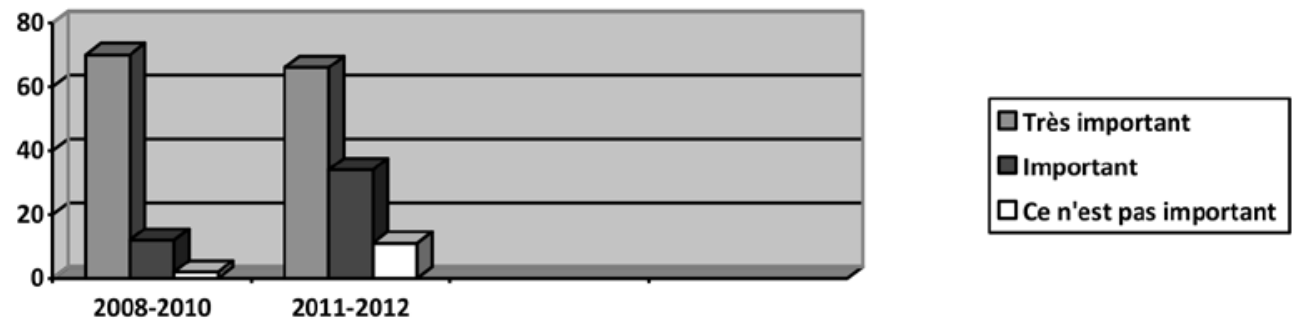

Graphique $^{\circ}$ 3. Importance des acquis linguistiques au travail (2008-2012)

Les apprenants apprécient plus l'apprentissage hybride car celui-ci s'appuie sur l'approche actionnelle liée à la vie professionnelle du douanier : gestion de l'emploi du temps d'un douanier, collaboration avec les collègues francophones, code d'éthique et de conduite des agents des douanes, contrôle douanier, comportement exemplaire, mission douanière à l'étranger, comparaison des douanes de Lituanie et de France, etc. L'approche hybride correspond parfaitement aux processus d'intégration européenne et de mondialisation qui ont demandé de réagir plus vigoureusement et efficacement dans le domaine d'études du plurilinguisme et de la didactique des langues étrangères.

En 1971, le Conseil de l'Europe a fixé deux principaux objectifs pour les langues vivantes (Trim, 1999 : 15-17) : 1) faciliter la mobilité des personnes en Europe grâce aux compétences lingustiques ; 2) créer des modules séparés en fonction de l'orientation scolaire ou professionnelle et des objectifs d'apprentissage. La communication officielle " Plan d'action sur l'apprentissage des langues et la diversité linguistique entre 2004 et 2006 » de la Commission de l'Europe a de nouveau souligné l'importance de la politique du plurilinguisme. Ce document a engagé les pays européens à respecter et à préserver la diversité linguistique pour prévenir et empêcher la discrimination basée sur la langue, à améliorer $1^{\prime}$ 'enseignement et les méthodes d'apprentissage afin de créer un environnement et des conditions favorables pour les langues vivantes.

\section{Multilinguisme en Lituanie}

En Lituanie, les langues les plus populaires sont le lituanien, l'anglais, le russe, l'allemand et le français. Selon les données du Département de statique de la République de Lituanie ( $\mathrm{cf}$. Tableau $\mathrm{n}^{\circ}$ 1), la langue choisie par les élèves le plus souvent est l'anglais, ensuite - le russe, l'allemand et finalement le français. Le statut de l'anglais augmente chaque année, tandis que celui du français et de l'allemand diminue en devenant soit la deuxième soit la troisième langue étrangère : 
Tableau $\mathbf{n}^{\circ}$ 1. Place du français dans les écoles de Lituanie en 2017-2018

\begin{tabular}{|l|c|c|c|c|c|c|}
\hline & $\begin{array}{c}\text { 1ère langue } \\
\text { étrangère }\end{array}$ & $\%$ & $\begin{array}{c}\text { 2ème langue } \\
\text { étrangère }\end{array}$ & $\%$ & $\begin{array}{c}\text { 3ème langue } \\
\text { étrangère }\end{array}$ & $\%$ \\
\hline Anglais & 287,218 & 98,86 & 1,108 & 0,77 & 0 & 0 \\
\hline Russe & 723 & 0,25 & 114,676 & 78,8 & 490 & 27,54 \\
\hline Allemand & 1,929 & 0,67 & 21,999 & 15,1 & 368 & 20,69 \\
\hline Français & 681 & 0,23 & 7,017 & 4,8 & 412 & 23,16 \\
\hline Autre & 0 & 0 & 745 & 0,51 & 509 & 28,61 \\
\hline
\end{tabular}

Le niveau du multilinguisme augmente en Lituanie. Le dernier recensement de la population lituanienne a démontré que le pourcentage des habitants maîtrisant deux langues avait augmenté de 3,9 pourcents : $29 \%$ en 2011 et $25,1 \%$ en 2001 (cf. Graphique $\mathrm{n}^{\circ} 4$ ), trois langues - de 1,6 pourcents : $6,6 \%$ en 2011 et $5 \%$ en 2001 , quatre ou plus de langues - de $0,5 \%: 1,3 \%$ en 2011 et $0,8 \%$ en 2001 sur l'échelle générale où 78,6 pourcents maîtrisent au moins une langue étrangère (ibidem) :
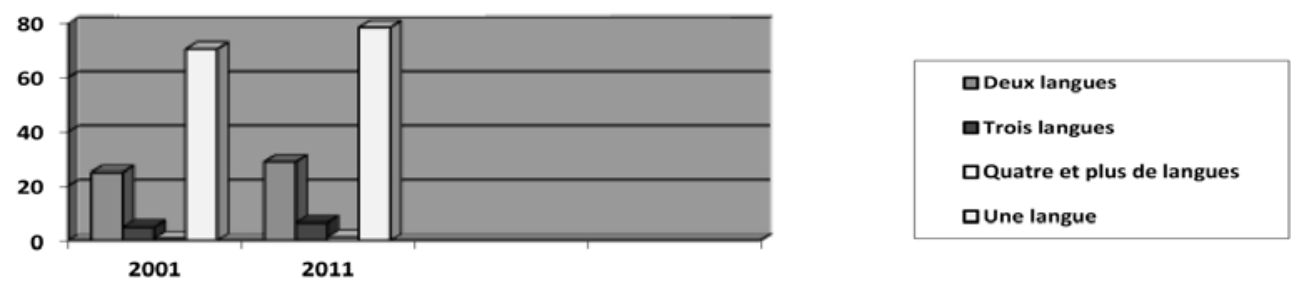

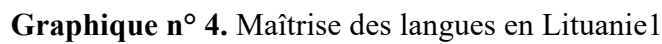

Les données d'Eurobaromètre sont encore plus encourageantes concernant le plurilinguisme en Lituanie : selon les enquêtes menées en 2012, la population de Lituanie est plus compétente au niveau linguistique que la moyenne de l'UE. D'après ce sondage, ce n'est que 8 pourcents de Lituaniens qui ne parlent aucune langue étrangère, alors qu'en Europe, c'est 46 pourcents ; 92 pourcents parlent au moins une langue étrangère et $54 \%$ en Europe, deux langues - 52\% en Lituanie et $25 \%$ dans l'UE, trois langues $-18 \%$ et $10 \%$ (ibidem).

Les données d'Eurobaromètre démontrent l'importance de l'allemand : 28 pourcents de Lituaniens considèrent la langue allemande la deuxième langue étrangère après l'anglais (ibidem). Pourtant, les données statistiques et la vraie situation de l'apprentissage des langues vivantes en Lituanie ne sont pas les mêmesentre 2010 et 2011, cette langue s'est retrouvée en 4ème position après l'anglais, le russe et le français (ibidem). A l'Académie, par ex., elle n'a pas été enseignée entre 2013 et 2015 en raison du manque d'étudiants désirant l'apprendre. C'est une 
situation paradoxale car d'un côté, l'Allemagne, avec laquelle la Lituanie a des relations historiques, économiques et culturelles assez fortes, est le pays le plus puissant dans l'UE et de l'autre côté, sa popularité diminue. La même situation est avec le français dont la présence dans les écoles et universités lituaniennes baisse aussi. Cette langue a toutes les composantes nécessaires pour la motivation : présence sur l'arène de la politique internationale (langue officielle de toutes les plus grandes organisations internationales), richesse culturelle et économique. Cependant, le français et l'allemand cèdent leurs places au profit d'autres langues.

\section{Préférences linguistiques des étudiants lituaniens}

Pour comprendre et évaluer le choix linguistique des étudiants de l'AML, le département des langues étrangères a mené une étude via les questionnaires anonymes distribués et analysés en 2015.107 personnes (65 cadets et 45 officiers) ont été interrogés par les enseignants du département. L'enquête comportait ces questions : «Pourquoi apprenez-vous l'anglais ? », « Pourquoi apprenez-vous le russe ? ». Il y avait ces options : « Je le veux », « On me le demande », « Je ne sais pas. J'en aurai peut-être besoin ». Les apprenants de l'AML ont été aussi demandés d'expliquer brièvement la raison et la motivation d'apprentissage.

Notre étude a montré que la majorité apprenait l'anglais parce qu'ils le voulaient -83 personnes (soit 78\%), 21 apprenants (20\%) - parce qu'on le demandait, et ce n'est que 3 personnes (2\%) qui ont dit qu'ils ne savaient pas pourquoi (cf. Graphique ${ }^{\circ} 5$ ). Ainsi peut-on conclure que la plupart d'apprenants d'anglais comprennent la nécessité de l'apprentissage ayant une grande motivation d'apprendre grâce aux réserves intérieurs. Tous les résultats sont présentés cidessous :

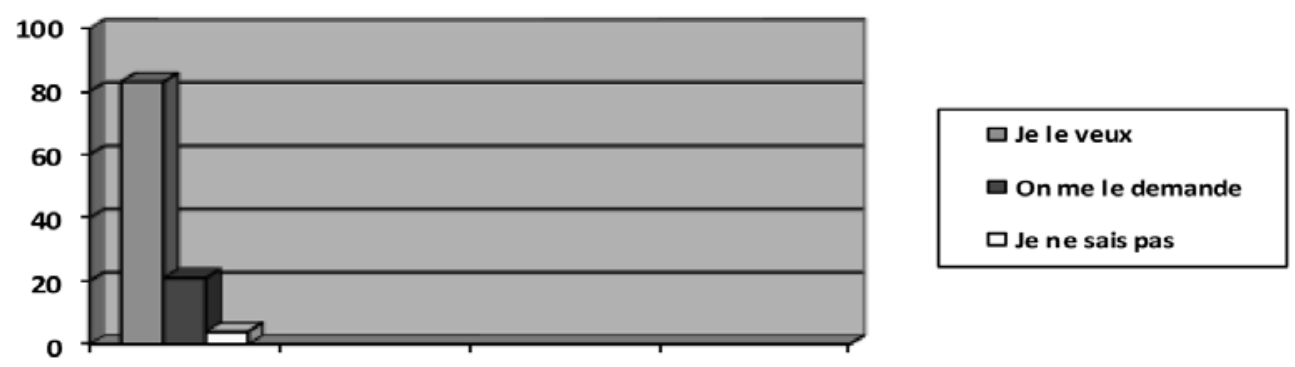

Graphique $\mathbf{n}^{\circ}$ 5. Apprentissage de l'anglais à l'AML

Ce n'est donc que 22\% d'apprenants (ibidem) qui sont motivées d'étudier l'anglais vu des facteurs extérieurs alors que les autres sont régis par des motifs intérieurs. 
Les résultats de l'étude sont différents concernant l'apprentissage d'autres langues (allemand, français et russe). Il s'est avéré que l'intérêt à apprendre les autres langues vivantes (surtout l'allemand et le français) était beaucoup plus faible. Cependant, les réponses et les explications concernant la motivation n'ont pas démontré une attitude négative des apprenants de l'AML envers ces langues. La plupart des personnes interrogées ont dit qu'ils n'avaient pas compris les avantages des cours et qu'ils ne voulaient pas être surchargés par des informations inutiles. De plus, la grande majorité ne voyait pas d'utilité pratique d'apprendre car, selon eux, il suffisait de maîtriser l'anglais et le russe pour se sentir bien et pouvoir réaliser leurs ambitions professionnelles ou évoluer dans la profession militaire. D'autres personnes ont avoué que les cours de français ou d'allemand donnaient très peu d'ECTS (N.B. 3 ECTS) pour leurs études et qu'ils n'avaient pas assez de temps pour ce type de cours supplémentaires. Il y avait aussi une petite partie de ceux qui avaient accusé les professeurs de l'AML ayant chargé leurs cours par trop de règles de grammaire que les cadets apprenaient seulement pour recevoir une bonne ou très bonne note qui leur permettait de toucher une bourse plus élevée.

Quant aux réponses concernant la langue russe, devenue la deuxième langue étrangère enseignée et étudiée à l'AML, 96\% de personnes interrogées ont souligné son importance dans la profession ou la vie. A noter, $51 \%$ d'étudiants l'apprennent car c'est la langue d'un ennemi et $37 \%$ pensent que c'est la langue d'un grand voisin alors que ce n'est que $12 \%$ qui croient qu'il suffit de maîtriser uniquement l'anglais et le russe pour communiquer dans le monde entier ou réussir. On voit tous ces résultats dans le sixième graphique (cf. ci-dessous) :
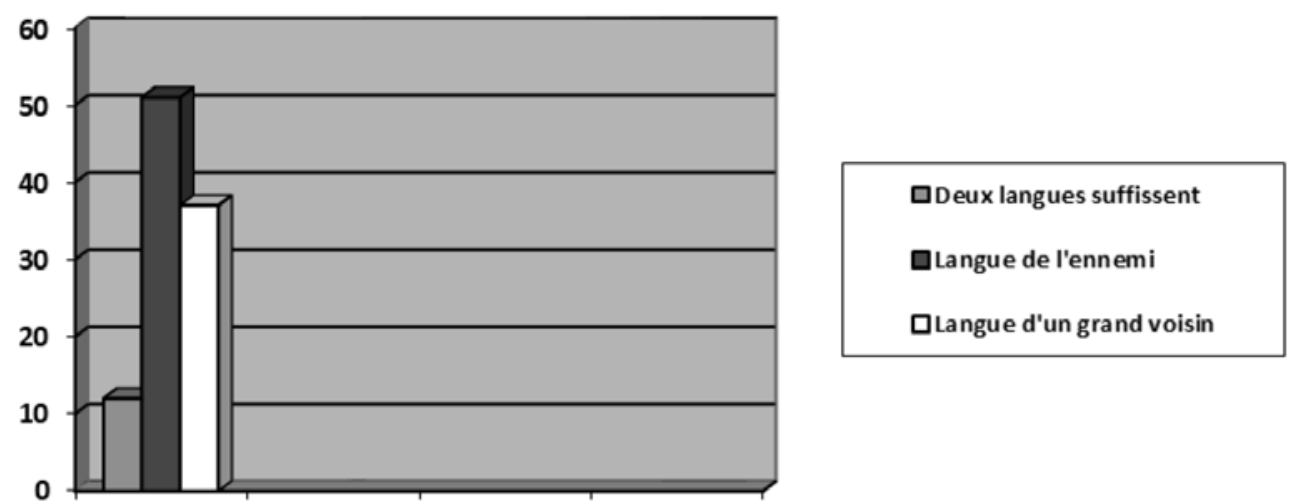

Graphique $\mathbf{n}^{\circ}$ 6. Apprentissage d'autres langues à l'AML

Il y a une bonne corrélation entre toutes les informations statistiques de l'AML et celles du Département de Statistique de la République de Lituanie concernant la maîtrise des langues étrangères : 


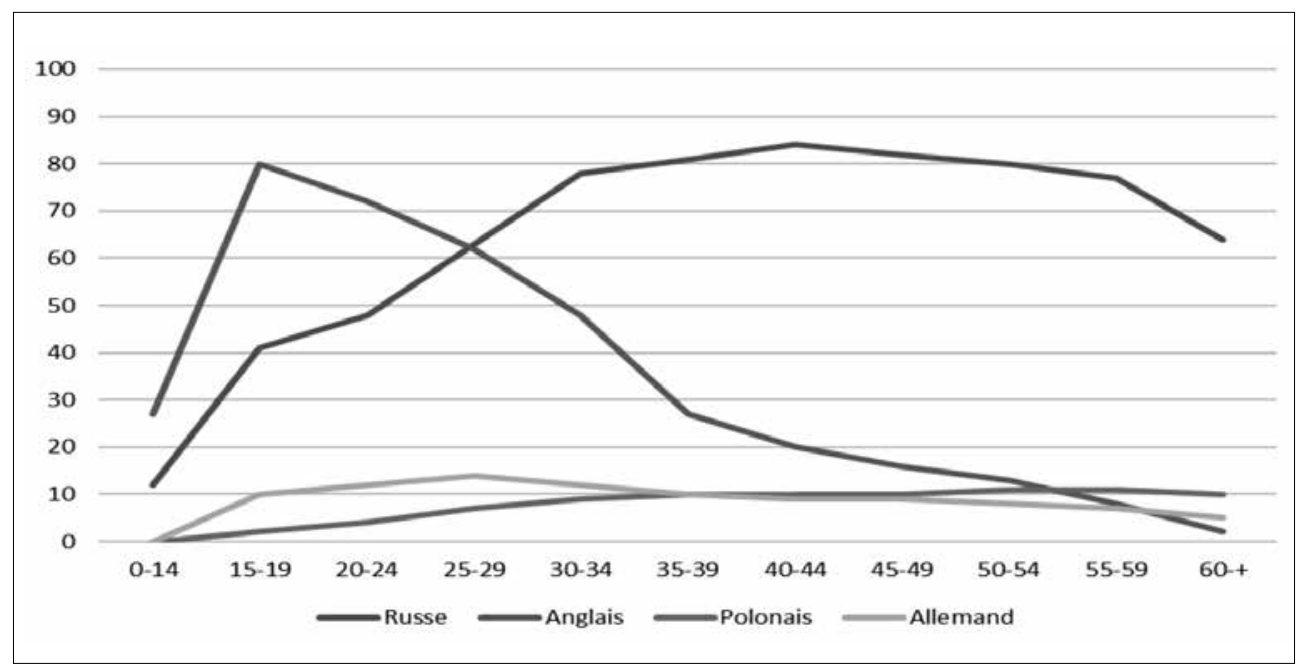

Graphique $\mathbf{n}^{\circ}$ 7. Maîtrise linguistique et âge 2

On revoit que les langues les plus populaires sont l'anglais, le russe et l'allemand (cf. Graphique $\mathrm{n}^{\circ} 7$ ). On observe également que l'âge le moins touché par l'apprentissage ou la maîtrise linguistique est celui des enfants jusqu'à 14 ans soit celui des personnes âgées plus de 60 ans (ibidem). Selon le Département de Statistique, la quantité des Lituaniens maitrisant une langue étrangère a augmenté de $70,6 \%$ en 2001 jusqu'à $78,5 \%$ en 2011 . La maîtrise de l'anglais a également grandi à partir de $16,9 \%$ jusqu'à $30,4 \%$, le russe est maîtrisé par $63 \%$ de citoyens, le polonais $-8,5 \%$, l'allemand $-8,3 \%$.

Les données du Département de Statistique nous indique de même l'âge maîtrisant le plus de langues, c'est celui des jeunes entre 20 et 35 ans qui maîtrisent deux ou trois langues (ibidem), c.-à-d., les étudiants sont les plus ouverts vers les langues vivantes. Or, le français y est absent. Cela nous suggère l'idée qu'il y a peu de francophones en Lituanie en prenant en considération l'importance de la langue française dans le monde entier où le français occupe la 5ème place parmi les langues les plus parlées avec 274 millions de locuteurs (selon les statistiques de l'Organisation internationale de la Francophonie) ${ }^{3}$. L'enseignement et l'apprentissage du FLE suit celui de l'anglais. En ce qui concerne le monde des affaires et celui d'Internet, cette langue est la 3ème dans le business et la 4ème dans le réseau mondial de communication.

2 cf. Les données statistiques viennent du site: https://osp.stat.gov.lt/documents/10180/217110/ Gyv+pagal+išsilavinima_ir_kalbu_mokejima.pdf/b75c5e7a-e733-48fa-95e4-83d485c6726a

3 cf. Les données statistiques viennent du site: https://www.francophonie.org/274-millions-defrancophones-dans.html 


\section{FLE dans les universités lituaniennes}

Il serait donc utile d'examiner le choix linguistique des étudiants lituaniens concernant leur préférence linguistique et la motivation d'apprendre le français. Les résultats de l'enquête, menée parmi 90 étudiants des facultés et universités différentes de Lituanie, offrent la possibilité d'identifier de nombreux facteurs. Les participants du sondage avaient entre 20 et 30 ans. L'enquête a été faite sur la base d'un site internet « ManoApklausa.lt » sous forme de questionnaire, fait et envoyé par les étudiants de l'université de Vilnius à leurs camarades ou connaissances, qui devaient répondre à 21 questions. Dans l'enquête, on trouve des questions comme : «Avez-vous déjà appris le français à l'école ? Avez-vous étudié le français à l'université ? Y avait-il la possibilité d'apprendre le français ? Pourquoi n'avezvous pas appris le français ? Quelles autres langues voudriez-vous apprendre ? Pensez-vous que la langue française soit utile dans votre avenir ? Estimez-vous le français comme une langue importante pour les générations à venir? ».

Le but de cette enquête était d'analyser pourquoi la langue française était choisie par les étudiants lituaniens et ce qui les motivait d'apprendre la langue qui n'est pas parmi les langues les plus populaires. L'analyse qualitative des données statistiques présente ainsi ces résultats (cf. Graphique $\mathrm{n}^{\circ} 8$ ) : premièrement, plus que la moitié des étudiants $(65,6 \%)$ - avaient la possibilités d'apprendre la langue française à l'école ou à l'université en préférant cependant d'autres langues comme l'anglais, le russe et l'allemand ; or, certains étudiants $(14,1 \%)$ voulaient apprendre la langue française à l'université, mais il n'y avait pas de possibilité ; deuxièmement, presque la moitié d'étudiants pensent que la langue française n'est pas populaire en Lituanie car ils ne voient pas d'environnement francophone où cette langue serait utile, même si beaucoup d'entre eux sont convaincus que la langue française est un atout pour les voyages ou le travail; troisièmement, la plus grande partie des sondés $(65,6 \%)$ sont de l'avis que la langue française est très importante pour la future génération étant employée dans le monde entier.

Les résultats de l'enquête n'ont pas illustré que les possibilités d'apprendre le FLE en Lituanie mais ont aussi clarifié les raisons les plus importantes d'apprentissage. Les motifs comme un bon travail avec un salaire élevé ainsi que la possibilité de vivre ou étudier dans un pays francophone sont les plus fréquents avec $94 \%$ de réponses (ibidem) : 


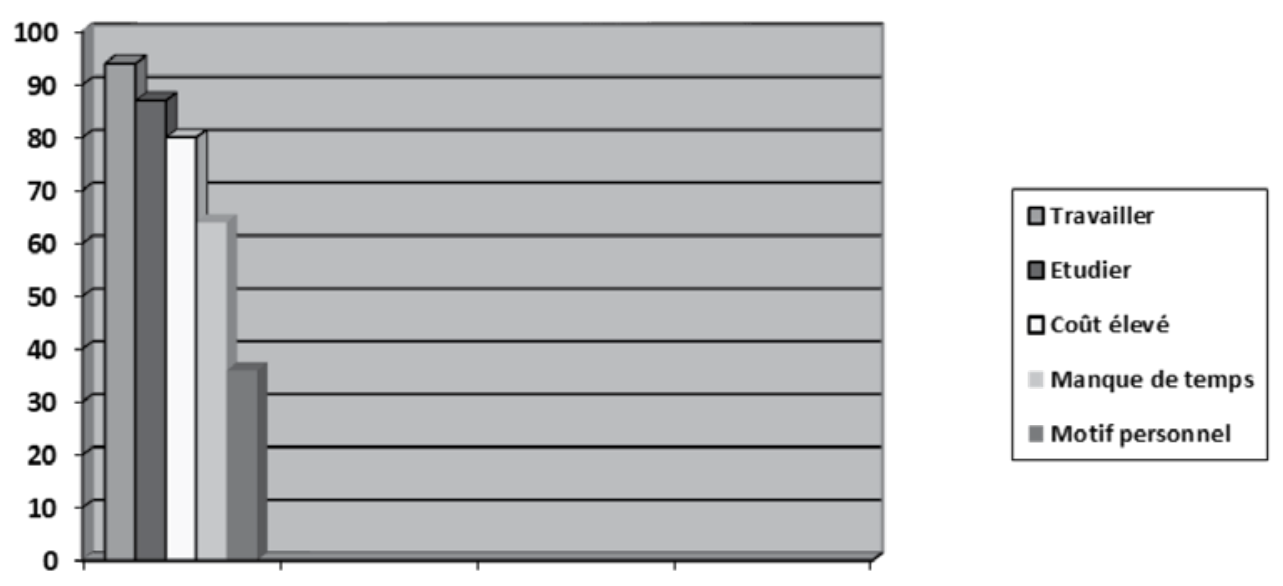

Graphique $\mathbf{n}^{\circ}$ 8. Motifs du FLE dans les universités

D'autres réponses $(80 \%)$ parlent d'un coût parfois trop élevé pour les étudiants désirant apprendre cette langue à l'Institut français ou chez les particuliers. Le manque de temps a été indiqué par $64 \%$ des répondants comme un très gros problème. A noter que beaucoup d'interrogés pensent qu'un homme français pourrait les motiver plus à apprendre cette langue assez difficile puisque la majorité des réponses appartenaient aux femmes qui choisissent le FLE plus souvent que les hommes ( 71 femmes et 19 hommes). La raison principale est donc le travail et la vie quotidienne. Ce sont deux facteurs principaux qui motivent les étudiants à apprendre le françias. Les critères comme le prestige, la popularité, l'offre institutionnel ou la politique linguistique officielle sont moins importants pour la motivation et la volonté d'apprendre.

\section{Réussite de l'apprentissage}

Pour apprendre une langue étrangère, il faut certainement être motivé. La motivation a fait l'objet de multiples recherches en psychologie et en sciences de l'éducation. Ce terme, commencé à être largement employé dans l'éducation à partir du milieu du siècle passé (Tavris et al., 1999 : 22), provient du verbe latin movere qui signifie "mouvoir, bouger", c.-à-d., la motivation est étroitement liée au concept de la croissance qui explique l'action d'un individu. C'est le facteur de la disposition affective d'apprendre. Le chercheur lituanien Jovaiša L. traite la motivation comme « [...] un processus psychophysiologique qui met en relation l'activité d'une personne et ses relations avec l'environnement en se basant sur un échange réciproque » (Jovaiša, 2007 : 172). Butkienė G. et Kepalaitė A. mettent ce critère du processus d'apprentissage à côté de l'agitation ou de l'excitation du comportement humain liée à la psyché individuelle (Butkienè, Kepaitè, 1996); Gage N. L. et Berliner D. C. l'expliquent comme des « [...] besoins, intérêts, valeurs, 
attitudes, aspirations et inclinations » (Gage, Berliner, 1994 : 263) ; Dornyei y voit un processus provoquant « [...] une grande force qui stimule une activité humaine jusqu'à ce que notre but soit atteint, même au moment lorsqu'une autre force tente de l'éliminer» (Dornyei, $1998: 121)$.

La motivation est également associée à l'atteinte des objectifs : « La motivation constitue donc un terme générique qui caractérise la nature de la force qui produit un comportement ou une action » (Masson, 2011:19). C'est l'énergie qui nous aide à gagner : " On peut aussi situer la motivation comme une intention de faire qui est reliée à une activation, une dynamisation psychologique, une énergie potentielle, prête à être mise en oeuvre ou à être déployée, mais qui peut aussi ne pas l'être si l'activité ne correspond pas aux attentes et aux souhaits» (Lemoine, 2008: 403). Petty G. a distingué sept principes de réussite : 1) croire à l'importance de l'apprentissage; 2) avoir confiance en ses propres forces ; 3) considérer le processus d'apprentissage comme un défi personnel ; 4) communiquer ; 5) compter sur l'aide de l'enseignant pour structurer et comprendre ; 6) respecter les délais ;7) apprendre plus les aptitudes ou les compétences et moins le contenu (Petty, 2009).

Les représentants du constructivisme social Williams M. et Burden L. R. divise la motivation en facteurs internes, liés aux approches affectives, cognitives et métacognitives de la compréhension traitée par la psychologie, l'éducologie ou la biologie ainsi qu'en facteurs externes, recherchés et étudiés en premier lieu par les sciences sociales (Williams, Burden, 1997). Ces facteurs sont propres également à la motivation d'apprendre une langue étrangère, ce qui mérite d'être discuté plus en détail.

On veut et on peut apprendre une langue vivante si on est motivé. Sans motivation, aucun processus cognitif, qui nécessite une réflexion, une mémorisation, une compréhension et une concentration, n'est possible. Il a été déterminé que la corrélation entre l'intelligence générale et la motivation d'apprentissage fluctue habituellement d'environ 0,50 et même de 0,80 (Rheinberg et al., 2000). La corrélation élevée entre les résultats d'apprentissage, la motivation et les compétences cognitives est souvent considérée comme l'un des arguments les plus significatifs pour la mise en valeur des recherches dans le domaine.

Sur cette base, on peut affirmer qu'il existe une vision unifiée des scientifiques dans la formulation des trois principaux facteurs de motivation dans l'apprentissage des langues : l'intention humaine bien déterminée, les efforts et l'énergie ciblés pour réaliser des objectifs linguistiques fixés (ibidem). Assurément, les résultats d'apprentissage seront encore meilleurs si l'apprenant a une attitude positive vis-àvis de l'apprentissage sans oublier, bien sûr, les capacités intellectuelles. C'est ainsi que Gardner R. C., l'un des scientifiques les plus éminents dans le domaine et le fondateur de la théorie de la motivation, décrit la motivation comme des efforts pour atteindre un objectif linguistique et une bonne attitude en face de l'apprentissage des langues (Gardner, Lambert, 1972).

Ce sont l'expérience individuelle, les résultats acquis, la réussite et les 
intérêts personnels qui influencent la motivation de chacun d'apprendre une langue étrangère (Berndt, 2002). Chaque apprenant adulte a son propre système d'apprentissage en s'appuyant sur son expérience et évolution linguistique. Ceux ou celles dont les souvenirs et le bilan personnels, liés à l'apprentissage, sont positifs ont des ressources intérieures plus élevées que ceux ou celles dont l'expérience est négative. Plus l'expérience est réussie, plus il est facile d'apprendre une ou des langues étrangères. Cela renforce et accélère l'apprentissage en rendant le processus éducatif plus significatif, intéressant et divertissant. Sans doute, l'apprenant n'est pas motivé grâce uniquement à ses ressources intérieures.

\section{Méthodes actives}

Les enseignants ont plusieurs charges aujourd'hui : enseigner, consulter, animer, entraîner, etc. Ils doivent aussi offrir aux adultes un enseignement de qualité. Ces derniers temps, on se concentre surtout sur la formation professionnelle des andragogues ainsi que sur l'approfondissement de leurs compétences, discutées largement parmi les scientifiques étrangers (Knowles, 1990; Malglaive, 1990) et lituaniens (Juozaitis, 2008). Dans un contexte actuel de multilinguisme et d'interdisciplinarité avec la diffusion massive des informations et le monde de plus en plus globalisé se trouvant aux prémices d'une quatrième révolution industrielle, il existe un large choix de méthodes qui permettent de développer l'autonomie de 1 'apprenant, de stimuler une pensée et une participation actives de tous les acteurs du processus éducatif.

Les pédagogues et andragogues ont utilisé très longtemps des méthodes traditionnelles, par exemple, la méthode de grammaire-traduction ou la leçon. Ces méthodes sont efficaces pour présenter des sujets inconnus parce qu'elles nécessitent une écoute active pour comprendre un nouveau sujet ou thème. Cependant, l'enseignement/apprentissage, qui ne s'appuie que sur des méthodes traditionnelles, devient monotone et surtout inefficace, éloigné souvent des actualités et des réalités du monde multiculturel car le niveau le plus élevé cognitif, où l'apprenant analyse et évalue, y est absent. Or, il vaut mieux se servir des méthodes et des formes d'éducation nouvelle ou moderne afin d'attendre les meilleurs résultats (Paulson \& Faust, 1999).

Les vecteurs des méthodes de l'apprentissage actif sont l'interaction et la discussion qui reflètent la vie moderne et s'appuie sur l'actualité. Ces méthodes incluent aussi des outils didactiques comme les jeux, la collaboration, les recherches (individuelles ou communes) de solutions, les projets communs ou individuels, l'expérience, la créativité, etc. Avant de présenter un nouveau sujet ou thème, on peut proposer quelques affirmations à discuter en provoquant ainsi une discussion ou des hypothèses parfois contradictoires. L'objectif recherché est de générer des idées et de recueillir des opinions personnelles ainsi que de connaître le niveau des apprenants ou leurs lacunes à combler. 
L'enseignant aide également l'apprenant à évaluer son niveau et état de préparation, ses difficultés et qualités, à exprimer son opinion personnelle. On peut commencer par des questions ou phrases provoquant le débat. On peut, par exemple, soumettre une liste de mots illustrant la règle de la distinction entre le genre masculin et féminin grâce à la connaissance des terminaisons : « un fromage, un paysage, un courage » mais " une image, une plage, une cage ». On peut également présenter avancer une affirmation contestable et demander qu'elle soit discutée : par exemple, on demande d'élaborer la liste des raisons de la dégradation des valeurs culturelles, sociales et familiales. Les apprenants échangent sur le sujet par groupe de deux ou trois et s'expriment, s'opposent ou approuvent. Le professeur note au tableau les propos des apprenants. La discussion achevée, chaque groupe présente au moins une idée. Les hypothèses les plus intéressantes et les plus originales sont évaluées, corrigées par l'enseignant si nécessaire au tableau ou sur l'écran interactif. Le temps des débats varie en fonction du niveau des apprenants, de la difficulté de la tâche ou de l'intérêt manifesté par les apprenants pour le thème.

L'enseignant ne doit pas évaluer ou critiquer les apprenants ni leurs propos, même s'il n'est pas d'accord. Son but est de provoquer l'intérêt des apprenants (surtout dans le cas des adultes) pour le sujet traité et de les amener à la recherche commune d'une réponse. La tâche terminée, l'enseignant y ajoute des commentaires en se basant sur ses connaissances théoriques et son expérience professionnelle liées au sujet traité en faisant ainsi des corrections nécessaires grammaticales, lexicales s'il s'agit d'un thème grammatical ou lexical ou encore sur les visions du monde si c'est un sujet d'actualité, en passant ensuite à une explication ou une présentation plus détaillée.

Les andragogues et professeurs, passants du paradigme de l'enseignement au paradigme d'apprentissage actif, saisissent les principes de base de l'éducation moderne où la compréhension de l'apprentissage individuel est primordiale. On apprend en se basant sur notre expérience, nos compétences, notre propre stratégie d'apprentissage. Les enseignants doivent partir des centres d'intérêts des apprenants afin de susciter l'envie d'explorer, d'apprendre et de coopérer. Donc on choisit des exercices très variés pour contenter les besoins personnels des apprenants.

Selon Howard Gardner et Thomas Hatch, les moyens non linguistiques de la communication (kinésique, proxémique, logique, musique, rythme, etc.), la dimension métalinguistique du langage, la perception spatiale et les relations interpersonnelles permettent de mieux apprendre (Gardner \& Hatch, 1989). Le processus traditionnel se limite à l'acquisition des compétences purement linguistiques. Il faut employer un apprentissage actif qui prend en compte différents aspects du langage et des approches actionnelles. Si la personne est susceptible de percevoir une information par les yeux, voire la vision, on lui offre des images, des photos, des schémas, etc.; par l'audition - des discussions, des débats, des extraits sonores, etc.; par la tactile - des jeux ou des activités ludiques. 


\section{Mémorisation verbale}

L'apprentissage moderne se dirige vers la motivation des apprenants et vers leur participation active, comme on l'a déjà vu ci-dessus. Un autre facteur, qui influence l'apprentissage, c'est l'aptitude des apprenants à la mémorisation et au pouvoir de reproduire des informations apprises. Cette disposition cognitive peut être développée chez l'apprenant. Pour cela, il faut de l'attention, de la concentration, de la visualisation, de la régularité, des répétitions et des émotions . Ebbinghaus H. relate l'étude d'une langue étrangère dans un apprentissage perpétuel des mots ou des groupements de mots, voire des phrases, ainsi qu'un établissement permanent des relations entre eux (Ebbinghaus, 1980). Les similitudes de l'apprentissage sensori-moteur et moteur favorisent notre développement linguistique car les signaux auditifs et kinesthésiques linguistiques, qu'on reçoit lors du processus d'apprentissage, sont liés aux sons et mots prononcés. La recherche des rapports entre la matérialisation sonore des mots et leur signification y est ainsi primordiale, surtout chez les adultes, en posant un tas de questions sur la vie réelle et les objets qui nous entourent.

En parlant de l'apprentissage/enseignement des jeunes ou des adultes, les questions les plus fréquentes sont : « Quand devient-on adulte ? », « Est-ce que les méthodes d'apprentissage et d'enseignement des langues dépendent de l'âge ? ", « Est-ce qu'un(e) étudiant(e) est déjà une personne adulte ? » et ainsi de suite. Il est impossible de répondre sans ambiguïté à ce genre de questions puisque certains deviennent adultes déjà à l'école grâce à leur caractère et grâce à leurs capacités cognitives, alors que les autres restent irresponsables, immatures, insouciants et passifs, c.-à-d., enfants, jusqu'à la fin de leur vie. On distingue souvent ces étapes du développement personnel : début de l'adolescence (12-18 ans), fin de l'adolescence (18-22 ans), jeunes adultes (22-34 ans), adultes (34-60 ans), adultes âgés (60-75 ans), vieillesse (plus de 75 ans) (Beresnevičienè, 2003). Il est important que l'enseignant soit en mesure d'évaluer ses apprenants en fonction de leur âge en se fondant sur des combinaisons ou des systèmes variés, qui s'appuient en grande partie sur la capacité intellectuelle de retenir et de répéter.

Notre mémoire n'est pas qu'une condition préalable importante pour le renseignement mais c'est aussi l'une des fonctions les plus signifiantes de notre fonctionnement psychique sélectif. Les scientifiques ont constaté qu'après deux semaines de cours, les adultes ne retenaient que 5\% de ce qu'ils entendaient, $10 \%$ de ce qu'ils lisaient, $20 \%$ - de ce qu'ils entendaient ou voyaient. Ce pourcentage augmenterait jusqu'à $50 \%$ si la nouvelle information était pratiquée dans les groupes de discussion, jusqu'à $75 \%$ - si les apprenants l'employaient au travail et jusqu'à 90\% - si l'on faisait apprendre aux autres (Danaitis, Usovaite, 2010).

Le modèle de la mémoire contient trois composantes de base, selon Atkinson R. et Shiffrin R. M. : 
Tableau n². Modèle d'Atkinson-Shiffrin ${ }^{4}$

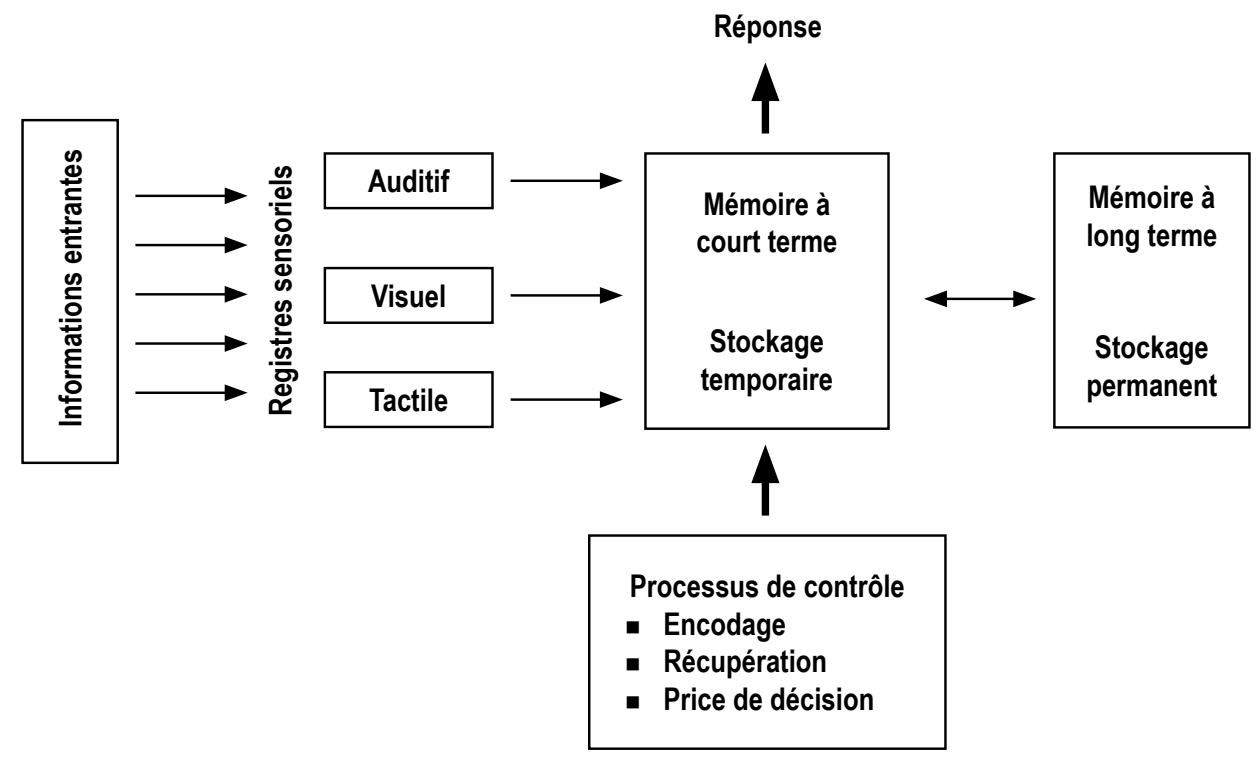

Ce modèle est utile pour nous grâce à la représentation significative de la répétition figurant dans le processus de contrôle (cf. Tableau n ${ }^{\circ} 2$ ) sous la forme de la récupération. Celle-ci est reliée tout d'abord à la mémoire à court terme en passant ensuite vers la mémoire à long terme. Cependant, la relation informative entre les deux types de la mémoire n'est pas forte ni stable. Ce qu'on garde dans la mémoire à long terme peut facilement revenir à son premier stade de la mémorisation. Par contre la répétition, basée sur la réflexion, l'analyse et la recherche des liens associatifs, peut nous servir dans le processus de la mémorisation à long terme.

Les recherches scientifiques ont démontré l'amélioration des capacités intellectuelles chez les apprenants grâce à la mémorisation du sens via les associations généralisées et systématisées (Jacikevičius, 1970). Ces associations reflètent les plus importants phénomènes et relations cognitifs faisant partie de la mémoire procédurale qui est une mémoire à long terme. En recherchant des rapports entre le sens, la représentation sonore ou visuelle, l'expérience personnelle ou commune, on crée des liens, systèmes ou groupes logiques linguistiques. C'est ainsi que la répétition devient plus productive et effective lors de l'apprentissage d'une langue étrangère. Les liens cognitifs diversifiés et systématisés permettent de renforcer la mémorisation en la rendant par conséquent meilleure, plus forte et plus rapide.

Le facteur de la rapidité, voire du temps, est très important pour l'apprentissage. Les scientifiques ont constaté le fait que le moment entre la perception et la réaction, la question entendue et la réponse prononcée, l'explication et la compréhension ne doit pas excéder à 0,5 seconde (ibidem). Sinon la mémorisation associative

4 http://www.reussir-en-universite.fr/apprendre-a-apprendre/elt_apprentissage3_3.html 
devient abusive et inefficace. Les expériences entre les humains et les animaux le démontrent - les deux perdent leur intérêt pour le processus d'apprentissage. Beaucoup de spécialistes de l'enseignement formel et non-formel sont d'accord avec le point de vue rappelant que le processus d'apprentissage nécessite la distinction de ces critères : a) besoin du savoir, b) autonomie, c) expérience, d) capacité d'apprendre, e) orientation, et f) motivation (Danaitis, Usovaitè, 2010).

\section{Activités théâtrales}

La mémorisation verbale est une partie intégrale des exercices d'origine théâtrale, comme le jeu de rôles ou la récitation des poèmes, car le théâtre nous oblige à apprendre et à savoir maîtriser notre attention en développant également notre imagination et nos émotions. A l'origine, le vocable "théâtre" est lié à la Grèce antique en signifiant "contempler, regarder". A l'époque moderne, quand la science et les arts devienennt de plus en plus polyvalents et pluridisciplinaires, le théâtre n'est pas associé qu'aux représentations publiques. La définition de l'art du théâtre a pris des proportions plus importantes et elle a dépassé les frontières d'un simple spectacle ou d'une pièce de théâtre.

Les activités théâtrales, au sein d'un cours ou d'une leçon, offrent la possibilité de faire évaluer l'individu (l'acteur se libère et s'enrichit mentallement et psychologiquement grâce à la découverte de sa propre personnalité); c'est aussi un moyen formidable pour recevoir de nouvelles informations théoriques (on lit, apprend, reproduit des mots, des textes, des situations, des histoires). Pour Robert Pignarre Pignarre (1991) et Bernard Dufeu (1996), les activités théâtrales permettent à l'apprenant de découvrire et de sentir plus facilement le rythme, l'intonation, la prononciation, la mélodie, la structure, la culture d'une nouvelle langue en s'exprimant avec sa propre voix et son corps, dirigé et guidé par l'andragogue ou le pédagogue.

Avant de commencer à jouer ou à interpréter des rôles et des textes, les apprenants doivent "s'échauffer" grâce aux exercices de bouche qui se concentrent sur la prononciation des voyelles, des consonnes, sur une intonation et une articulation, sur le débit de la parole le plus proche de la langue d'origine, etc. Dufeu propose, par ex., de donner aux élèves des virelangues faciles et drôles pour travailler le rythme et l'intonation : " Chasseur sachant chasser doit savoir chasser sans chien! Si ton tonton tond mon mouton, ton tonton tondu sera! Ce verrou bien verrouillé, le déverrouilleriez-vous? », etc.

Selon Dufeu, pour sentir et apprivoiser une mélodie correcte de la langue française, la mémorisation et l'interprétation des poèmes de J. Prévert serait idéale : « Un curé noir [aut. on monte la voix] sur la neige blanche [aut. on la baisse], c'est triste à voir [aut. on la monte] même le dimanche [aut. on la baisse] », etc. Afin de travailler sur la sémantique, on peut réciter des poèmes avec des gestes, par ex., le poème de Yan Marzin : «Il disait me voir [aut. on montre les yeux] mais il était aveugle [aut. on cache les yeux]. Il disait m'entendre [aut. on montre les oreils] mais il était sourd [aut. on cache les oreils]. Il disait me comprendre [aut. on met 
les mains au niveau du couer] mais il était trop intelligent [aut. on lève les mains en haut] ». Ou encore un autre exemple de l'exercice qui aide les apprenants à apprendre le français grâce au lien entre le geste et la parole : "' 'Oui' est blanc [aut. les paumes ouvertes tournées vers le haut]. 'Non' est noir [aut. les paumes ouvertes tournées vers le bas]. 'Oui' est noir [aut. les paumes ouvertes tournées vers le bas]. 'Non' est blanc [aut. les paumes ouvertes tournées vers le haut]».

L'objectif de tous ces exercices est de faire réagir, bouger, interagir les apprenants dans une langue étrangère pour leur faire acquérir du vocabulaire, de perfectionner leur accent, la prononciation. Après les exercices de l' "échauffement", on peut passer aux dialogues. On forme des groupes, on distribue des extraits et chaque groupe montre le travail fini à tour de rôle. Les extraits doivent faire nâitre un certain intérêt dans l'âme des apprenants pour la langue grâce au lien des dialogues avec leur vie personnelle ou professionnelle. Pour l'enseignement hybride (cf., ci-dessus), on utilise, par ex., les dialogues prises du module "Formation professionnelle combinée. Le français professionnel des douaniers, niveau B1 » („Kombinuotas mokymas. Muitinès pareigūnų prancūzų kalba, B1 lygis“"5).

Voici un extrait d'un tel dialogue, sur lequel les douaniers travaillent en formation professionnelle : «- Allo Irma, c'est Mindaugas. Alors cette installation à Paris, comment ça se passe ? - Oh, je ne suis là que depuis une semaine. J'ai passé mon temps à me présenter, rencontrer des gens... J'ai tout de même réussi à installer mon bureau. - Ce vieux bureau inconfortable ?! - Tu ne vas pas le reconnaître ! ». Ainsi, les apprenants connaissent à l'avance le contexte (un collègue qui a déménagé), le moment (une pause de déjeuner), ce que font les personnages (ils parlent au téléphone) et les rôles qu'ils tiennent (ce sont des collègues qui ne se sont pas vus depuis longtemps).

Comme ce travail se fait au début dans un petit groupe, cela pourra rassurer les personnes timides qui n'osent pas parler devant toute la classe mais qui pourront parler dans un petit groupe avec les autres en pratiquant le français. C'est ainsi que se passe la libération, l'ouverture et la connaissanse de sa propre personnalité grâce au français. Pour les activités théâtrâles, il est assez important d'essayer d'entrer dans la peau de quelqu'un d'autre et de collaborer avec les autres. On réussit donc à réunir deux grands objectifs didactiques : libérer son être afin d'affronter sa peur de parler une langue étrangère en public et retrouver son rôle social pour pouvoir interagir avec d'autres acteurs sociaux.

L'interprétation des extraits des pièces de théâtres ou la récitation des poèmes ne sont pas les seules activités théâtrales. Le théâtre moderne a transgressé ses frontières classiques pour s'ouvrir vers la pluridisciplinarité. Tout dépend de la créativité et de la volonté des enseignants ainsi que de la participation active des

5 http://www.cust.lt/mport/failai/struktura_ir_kontaktai/muitines_mokymu_centras/mokymo_ programos/kombinuoto_mok_programos/KOMB̈B_MUITINES_PAREIGUNU__PRANCUZU_ KALBA_B1_LYGIS_2010_05_31.doc 
apprenants. Cependant, si ces activités peuvent être au service de l'apprentissage/ enseignement d'une langue étrangère (voire du français), elles ont aussi des limites : réticence psychologique chez certains participants, manque de repères ou d'objectifs bien clairs et concrets pour les apprenants, efforts considérables pour créer ou maîtriser une ambiance favorable à l'apprentissage et à la créativité (surtout en travaillant avec les enfants), différences psychologiques et personnelles des apprenants, nécessité d'un contrôle ou d'une direction perpetuels pour les niveaux débutants, etc. Les activités théâtrales peuvent donc être considérées comme un appui non négligeable mais elles ne peuvent pas faire l'objet d'un système d'enseignement des langues étrangères autonome et indépendant.

\section{Conclusions}

Pour conclure, on peut dire que la politique linguistique de l'Union européenne devient de plus en plus homogène dans les pays européens. L'enseignement/ apprentissage du FLE est lié à la nécessité de parler plusieurs langues en Europe. Tout dépend des besoins d'apprentissage et de l'autonomie des apprenants, de leur expérience et de leurs styles de travail.

Le travail de l'enseignant du français consiste aujourd'hui à diriger le processus éducatif en se focalisant sur la formation des compétences linguistiques appropriées, sur l'interaction, sur le respect des autres et sur la formation de la pensée positive chez l'apprenant en soutenant ainsi sa motivation. La nature et la forme du processus éducatif sont en train de se modifier. Les enseignants perdent leur rôle de la principale source d'information. Les rôles et les responsabilités sont maintenant partagés entre tous les acteurs du processus éducatif.

Les principales tendances de l'enseignement du FLE sont liées à l'enseignement/ apprentissage actif qui est au service de l'augmentation de l'autonomie chezl'apprenant et de la motivation intérieure si importante pour l'apprentissage. Les approches didatctiques actives comme la formation professionnelle combinée, utile pendant des moments financièrement difficile car elle permet d'apprendre plus de gens avec des dépenses moins élevées, des activitiés théâtrales, utilisées par des enseignants motivés et créatifs, la verbalisation verbale, peu développée en Lituanie parce qu'elle nécessite une écoute ,participative" active de tous les acteurs du processus, sont importants mais ce sont des appuis supplémentaires pour rendre le moment d'apprentissage en français plus attractif et interactif en développant des compétences personnelles et professionnelles des apprenants liées à la culture francophone.

Les formes d'enseignement doivent être très variées afin d'inspirer et de soutenir l'intérêt pour la matière enseignée dans l'âme de l'apprenant et pour augmenter la motivation d'apprendre le FLE. La meilleure leçon ou un cours réussi, c'est quand l'enseignant du FLE se sent avoir laissé une partie de sa personnalité et l'élève - avoir trouvé la clé pour apprendre à se connaître. 


\section{Bibliographie}

1. Atkinson R. C., Shiffrin R. M. (1968). «Human memory: a proposed system and its control processes ». In Spence J. T. The psychology of learning and motivation (Volume 2). New York.

2. Beresnevičienè, D. (2003). Jauno suaugusiojo psichologija.Vilnius.

3. Berndt A. (2002). Motivation ist nicht statisch - Motivation ändert sich. In: Fremdsprache Deutsch 2/26. Bonn.

4. Butkienè G., Kepalaitė A. (1996). Mokymas ir asmenybès brendimas. Vilnius.

5. Danaitis K., Usovaite A. (2010). Medijomis paremtas déstymas kaip informacijos pateikimo ir įsisavinimo pagalbiininkas. Vilnius.

6. Dörnyei, Z., Ottó, I. (1998). Motivation in action: A process model. Working Papers in Applied Linguistics University of Nottingham.

7. Duffeu, B. (1996). Les approches non conversationnelles des langues étrangères. Paris.

8. Ebbinghaus H. (1980). Human Memory: Structures and Processes. San Francisco.

9. Gage N.L., Berliner D.C. (1994). Pedagoginè psichologija. Vilnius.

10. Gardner R. C., Hatch T. (1989). Educational Implications of the Theory of Multiple Intelligences. Educational Researcher, Vol. 18, No. 8, pp. 4-10.

11. Gardner R. C., Lambert W. (1972). Attitudes and motivation in second language learning. Rowley. Newbury House.

12. Jacikevičius A. (1970). Daugiakalbystès apybraiža. Vilnius.

13. Jovaiša L. (2007). Enciklopedinis edukologijos žodynas. Vilnius.

14. Juozaitis A. M. (2008). Andragogų praktikų neformaliojo mokymosi modelis. Vilnius.

15. Knowles, M. (1990). The adult learner. A neglected species, 4th Edition. Houston.

16. Lemoine C. (2008). «La motivation, notion fluctuante (faut-il être motivé pour travailler ?) », Carriérologie. Volume 11.

17. Masson, J. (2011). Buts d'accomplissement, Sentiment d'efficacité personnelle et intérêt. Université Paris Ouest Nanterre.

18. Minglang, Z. (2003). Multilingualisme in China -The Politics of Writing Reforms for Minority Languages 1949-2002. Mouton de Gruyter.

19. Paulson, R., Faust L. (1999). Active Learning for the College Classroom. Los Angeles.

20. Petty, G. (2009). Teaching Today a Practical Guide. From Nelson Thornes.

21. Pignarre, R. (1991). Histoire du théâtre. Paris.

22. Rheinb-erg, F., Vollmeyer, R., Rollett, W. (2000). Motivation and action in self-regulated learning. San Diego.

23. Rosen, E. (2010). Le point sur le Cadre européen commun de référence 
pour les langues. CLE international.

24. Tavris, C., et al. (1999). Introduction à la psychologie: les grandes perspectives. Editions du Renouveau pédagogique.

25. Trim, John L. M. (1999). Les langues vivantes au Conseil de l'Europe 1954-1997. Conseil de l'Europe. Strasbourg. 15-17.

26. Williams, M., Burden R.L. (1997). Psychology for Language Teachers: A Social Constructive Approach. Cambridge.

1 http://www.veidas.lt/tag/kalbos

2 https://osp.stat.gov.lt/documents/10180/217110/Gyv+pagal+išsilavinima_ir_kalbu_mokejima.pdf/b75c5e7a-e733-48fa-95e4-83d485c6726a

3 https://www.francophonie.org/274-millions-de-francophones-dans.html

4 http://www.reussir-en-universite.fr/apprendre-a-apprendre/elt_apprentissage3_3.html 


\title{
LEARNING FRENCH IN LITHUANIA AND MOTIVATION
}

\author{
Dr. Miroslav Stasilo \\ General Jonas Žemaitis Military Academy of Lithuania \\ Vilnius University
}

\section{Summary}

Europe has been characterized by multilingualism since the earliest times until now. Plurilingualism and multiculturalism is the basis and particularity of the European identity. The language policy of the European institutions occupies an important place in Europe. For example, in Lithuania, the number of persons mastering two or three foreign languages is higher than that in the European Union; however, the number of francophones is lower compared to the European average.

It is advantageous to examine the point of view of the Lithuanian students studying at the General Jonas Žemaitis Military Academy of Lithuania, Vilnius University and Customs Training Center about their motivation to learn French. Motivation is a decisive factor in the educational process. The description and analysis of the survey results related to learning French or other foreign languages in the aforementioned educational institutions revealed the main factors in the language choice of the Lithuanian students.

A modern teacher must first create favorable conditions for learning by helping a learner to wait for his goals. There is a wide range of methods and opportunities for learning French, for example, hybrid teaching, verbal memorization or theatrical activities. The main purpose of these methods is to encourage and support autonomy, logical thinking, integration and active participation in the social life of learners. The modern educational process envisages collaboration and active interaction between the teacher and the learner. The French teacher, based on active methods and creativity, must take into account the differences concerning motivation, level, experience, autonomy and individual objectives.

Keywords: multilingualism, French as a foreign language, motivation, active learning, blended learning, theatrical activities. 


\section{AUTORIAUS LYDRAŠTIS}

Autoriaus vardas, pavardè: Miroslav Stasilo

Mokslo laipsnis ir vardas: filologijos mokslų daktaras

Darbo vieta ir pareigos: Lietuvos karo akademijos Užsienio kalbų katedra; Vilniaus universiteto Filologijos fakulteto Taikomosios kalbotyros institutas

Autoriaus mokslinių interesų sritys: užsienio kalbos, sociolingvistika, politikos mokslai

Telefonas ir el. pašto adresas: + 3706 1144811; francaisprancuzas@yahoo.fr

\section{AUTHOR'S COVER LETTER}

Author's name, surname: Miroslav Stasilo

Academic degree and name: Doctor of Humanities

Workplace and position: General Jonas Žemaitis Military Academy of Lithuania, Department of Foreign Languages; Vilnius University, Faculty of Philology, Institute for Applied Linguistics

Author's research interests: foreign languages, sociolinguistics, political science

Telephone and e-mail address: +37061144 811;

francaisprancuzas@yahoo.fr 